\title{
A Simple Colorimetric Method for the Evaluation of Chitosan
}

\author{
Mohamed Abou-Shoer \\ Department of Pharmacognosy, Faculty of Pharmacy, Alexandria University, Alexandria, Egypt \\ E-mail:aboushoerm@yahoo.com \\ Received June 19, 2010; revised August 7, 2010; accepted August 12, 2010
}

\begin{abstract}
A simple sensitive and rapid colorimetric method has been developed, and herein described, for the qualitative and quantitative chemical assessment of the commercially available chitosan products. The described method relies on the reactivity of the basic amino function of chitosan with the acid dye bromocresol purple. The applied technique allows assessment of variability and selectivity changes in the quality of the marketed chitosan products.
\end{abstract}

Keywords: Chitosan, Dye, Colorimetric, Bromocresol Purple

\section{Introduction}

Chitosan is a natural biocompatible polymer derived from the naturally-occurring polysaccharide-based biopolymer, chitin, by deacetylation with an alkali leaving behind a free amino group (-NH2) (Figure 1) [1]. Chitosan naturally exists only in a few species of fungi but it is mainly extracted from the cuticular and exoskeletons of invertebrates like crustaceans, mollusks, crabs and shrimp. Crabs obtained from seafood processing waste are an important commercial source. Chitin is the second, after cellulose, most abundant naturally occurring polysaccharide. Chitin is composed of 2-acetamido-2-deoxy$\beta$-D-glucose units that are combined by 1-4 glycosidic linkages, forming long un-branched linear polymeric chains. Therefore, the biopolymer chitosan is composed of $\beta$-2-amino-2-deoxy-D-glucopyranose(glucosamine units) and $\beta$-2-acetamido-2-deoxy-D-glucopyranose [2]. Chitosan possesses unique properties like its ability to form films, and possesses a positive ionic charge which develops its ability to chemically bind with negatively charged fats, lipids and bile acids. Chitosan has a wide range of applications in diverse fields like in health (ranging from medical sutures to beauty aids), water purification (coagulants for waste treatment), biomedical applications, agriculture (seed coatings), biotechnology, nutrition (dietary supplements), and in the finishing process of textile fibers [3]. Chitosan is commercially available from many suppliers in various grades of purity, molecular weight, and degree of deacetylation. The de- gree of deacetylation is one of the most important chemical characteristics as it reported to influence the physicochemical properties and the performance of chitosan in many of its applications. Chitosan versatility depends mainly on the chemically reactive amino groups.

The degree of deacetylation of chitosan ranges from $56 \%$ to $99 \%$, depending on the crustacean species and the preparation method. Various methods have been reported for the determination of the degree of deacetylation of chitosan. These included ninhydrin test, linear potentiometric titration, infrared spectroscopy, nearinfrared spectroscopy, nuclear magnetic resonance spectroscopy, hydrogen bromide titrimetry, infrared spectroscopy, elemental analysis, colloidal titration, circular dichroism, ultraviolet spectroscopy, pyrolysis-gas chromatography, gel permeation chromatography and thermal analysis, acid hydrolysis, and X-ray diffraction methods and first derivative UV-spectrophotometry [46]. Chitosan could be also assayed colorimetrically using Cibacron brilliant red 3B-A [7,8].

All the above mentioned methods employed for the evaluation of chitosan slightly vary when measuring the number of free amino groups in the structure (deacetylation degree, DD). Obviously, the DD values are highly dependent on the analytical methods employed $[9,10]$. Hence, we propose that the analytical method used for the evaluation of chitosan products would also consider the functional capacity of the matrix. This approach is easily perceived when a reference matrix is taken into account. 


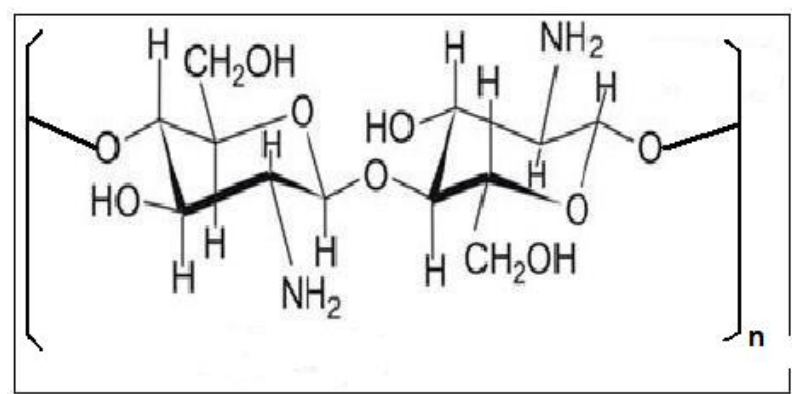

Figure 1. Structure of Chitosan unit.

Colorimetric assays are often developed as simple and sensitive practical procedures to rapidly evaluate drug quality in rapid and inexpensive protocol. Chitosan possesses a free amino group that acts as a reactive binding site for anionic dyes such as bromocresol purple to produce a color-bound complex. Therefore, our objective is to develop and evaluate a colorimetric technique to measure the functional capacity, which relates to deacetylation degree, so as to quantitatively assess these products in pharmaceutical preparations.

\section{Methods-Experimental Data}

\subsection{Reagents and Apparatus}

All reagents, hydrochloric acid, pyridine, acetic anhydride and sodium hydroxide, were of analytical-reagent grade, distilled or deionized water (DI) was used to prepare all aqueous solutions. Pharmaceutical-grade chitosan was used, bromocresol purple Indicator is a product of Reidel-DeHaen Ag, Seelze-Hannover, sodium bicarbonate, and sodium hydroxide from Sigma-Aldrich (St. Louis, MO, USA); Microgranular pre-swollen DEAE-32 Cellulose, Whatman, Springfield Mil, Madison, Kent, England.

\subsection{Apparatus}

Shimadzu UV 1601PC, UV/VIS double beam spectrophotometer (Kyoto, Japan) was used and equipped with 1 cm quartz cells and connected to IBM compatible computer. The software was UVPC personal spectroscopy software version 3.7 (Shimadzu). The spectral width applied was set at $2 \mathrm{~nm}$.

\subsection{Sample Preparation}

The colorimetric methods were evaluated in terms of linearity, precision, and accuracy by using different pharmaceutical batches of raw material compounds. Accurately weighed quantities of three commercially available (1-60 mgs) chitosan powder were introduced into small sintered funnel or filtration tubes. The chitosan sample were soaked with $0.2 \mathrm{ml}$ of water allow swelling of the polymer matrix.

\subsection{Bromocresol Purple Dye Solution}

Dye solution is prepared by dissolving $100 \mathrm{mg}$ of bromocresol purple in $5 \mathrm{~mL}$ of ethanol.

\subsection{Diethyl Amino Cellulose (DEA-32)}

Diethylamino cellulose ion-exchanger was used as a functional reference to relate the chitosan performance. DEAE cellulose was selected because of its structural resemblance to chitosan. Accurately weighed quantities (10-80 mgs) of DEAE cellulose ion exchanger were treated the same procedure like chitosan samples.

\subsection{Preparation of Acetylated Chitosan}

$200 \mathrm{mgs}$ from each chitosan samples were suspended in $0.5 \mathrm{ml}$ of dry and distilled pyridine. $2 \mathrm{ml}$ of acetic anhydride were added and stirred for 4 hours at room temperature then kept in dark for overnight. The reaction mixture is then quenched with cold water and the product, acetylated chitosan, was filtered off, washed with DI followed by ethanol and dried in an oven at $60^{\circ} \mathrm{C}$ for 15 minutes.

\subsection{Colorimetric Assay}

Bromocresol purple was used for the colorimetric assay and prepared at a concentration of $20 \mathrm{mg} / \mathrm{mL}$ in water. For all chitosan samples (weights from $1 \mathrm{mg}$ and up to 50 mgs of chitosan or their equivalent amounts) were accurately weighed. The chitosan samples were weighed either directly into small sintered glass funnels, or in Pasteur pipettes (disposable pipettes) packed at the bottom with glass wool. Secure the porosity of the filter pad, especially when glass wool, to carefully hold and do not allow passage of any of the loaded chitosan powder. The powdered sample in each tube was then wetted with 0.2 $\mathrm{mL}$ of DI water and allowed to soak for 15 minutes to allow possible swelling of the matrix. Approximately 0.3 $\mathrm{mL}$ of the dye solution is slowly passed through the sintered funnels. Each tube is then loaded with $0.2 \mathrm{ml}$ of the dye solution. Excess dye solution is drained out and excess dye was washed out with $0.5 \mathrm{~mL}$ of DI followed by $95 \%$ ethanol till complete removal of all color in the wash solution. The tube packed with chitosan-dye complex is then contained in clean $20 \mathrm{~mL}$ volumetric flasks. The chitosan-bound dye was then stripped off the bed by $20 \mathrm{ml}$ of $1 \mathrm{~N} \mathrm{HCl}$ solution and completed to volume. The acid solution is filtered through a $0.45 \mu$ membrane filter. Five milliliter aliquots were withdrawn from each sample concentration into a separate $50 \mathrm{~m}$ volumetric flask and 
completed to volume with $1 \mathrm{~N}$ sodium hydroxide solution. The developed blue color for each sample concentration is used for absorbance measurement at $589 \mathrm{~nm}$.

\subsection{Calibration Graphs}

$5 \mathrm{~mL}$ aliquots from the liberated sample solutions were transferred into $50 \mathrm{~mL}$ volumetric flasks. The reaction flasks are completed to volume with $1 \mathrm{~N} \mathrm{NaOH}$ and the solutions were measured spectrophotometrically at the $\lambda \max$ of $589 \mathrm{~nm}$ and the recorded absorbances for different samples of each sample were used to construct calibration graphs (Figure 2).

\section{Results}

The product from acetylating chitosan was treated with the dye solution the same way as described for chitosan. Although the acetylated chitosan matrix have acquired a red color once wetted with bromocresol purple, yet, the acidic solution used to drive out the adsorbed dye did not release any noticeable color intensity. Moreover, when the collected acidic wash solution was treated with sodium hydroxide and the measured at the same wavelength, such solutions did not record any appreciable absorbance readings. This finding illustrates that the presence of the free amino groups are essential for the working-efficiency of chitosan as an ion-exchanger. All chitosan samples, however, have produced abstracted appreciable quantities of the dye and released considerable color intensities upon acidification. The sensitivity of color-measurement is further enhanced by shifting the $\mathrm{pH}$ to the alkaline side with sodium hydroxide. The quantities of the dye freed from the chitosan-dye complex are then proportional to the quantities of chitosan used and hence calibration graphs were constructed. The good linearity and slope of the calibration graphs for the different chitosan batches indicate the efficient and strong sensitivity of the chitosan polymer in binding acidic compounds. Alternatively, when DEAE cellulose was treated with the dye solution, it showed a better linearity and a superior capacity. This data suggests that DEAE cellulose retains better functional characteristics that observed with chitosan polymers (Figure 3).

\section{Discussion}

The high value of the correlation coefficient and the intercept value were used to evaluate the linearity of the calibration curves. Regression analysis of these plots using the method of least squares had produced correlation coefficients (r) equal to 0.9904-0.998 indicating a good linearity (Figure 2). The sensitivity of the method to different batches is evident from the slope and intercepts values in the calibration curves. Moreover, the in- tercept and slope values of DEAE-cellulose in comparison to those obtained with chitosan products tip off the analyst to the performance properties, efficiency and capacity of such polymers as anionic exchangers relative to a different match of known operational parameters. The reported methods for the evaluation of chitosan products target measuring the deacetylation degree (DD) to reflect the quantity of free amino group on the chitosan matrix relative to the fully acylated chitosan (chitin). However, those methods do not gauge the factual capacity or working efficiency of the polymer. Accordingly, it has been viewed as more sensible to compare chitosan products as "functional" rather than chemical operators. Synthetic ion-exchangers of known chemistry, capacity

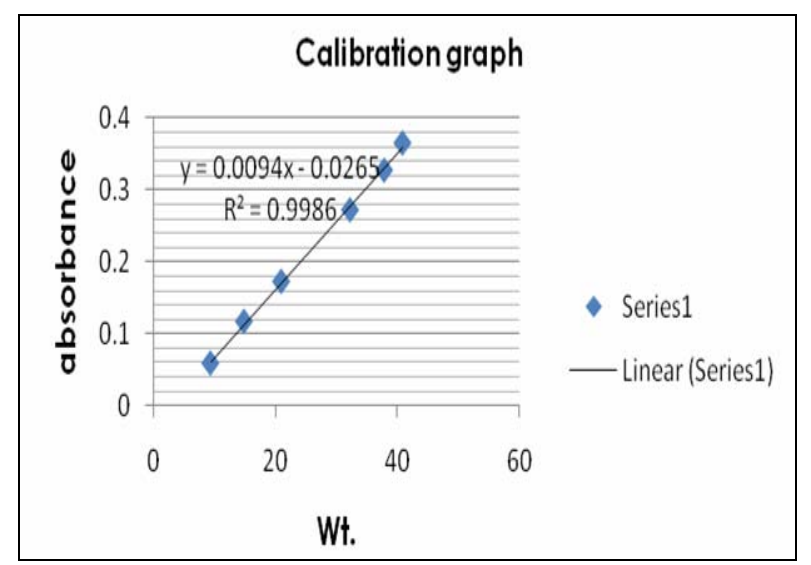

Figure 2 Calibration curve for chitosan.

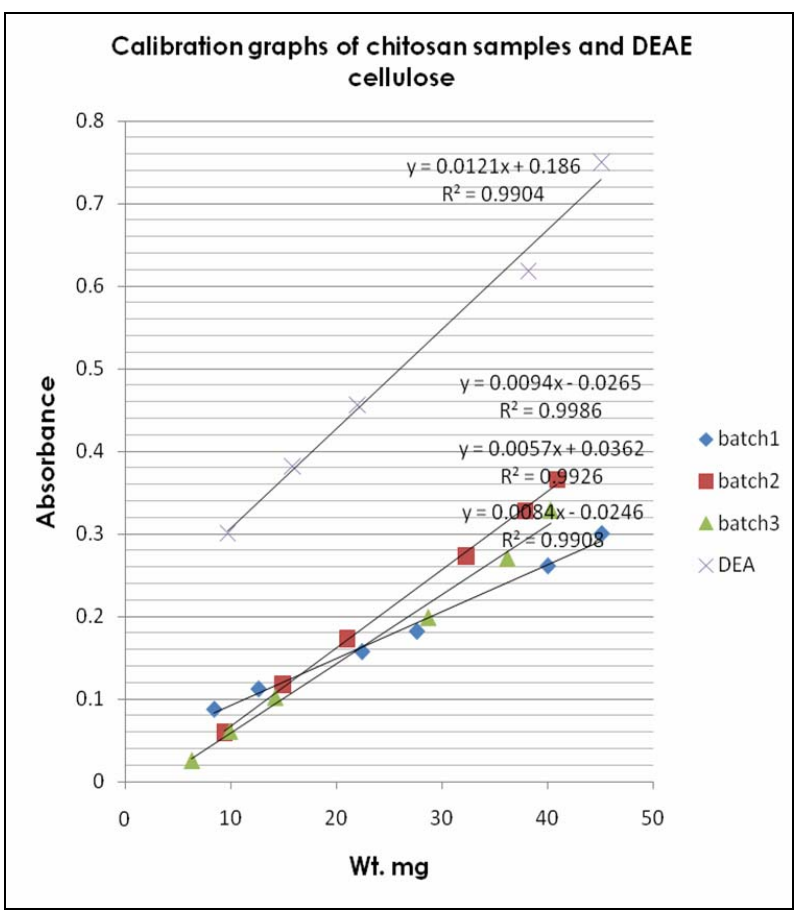

Figure 3 Comparison of chitosan samples and DEAE cellulose. 
and performance, like DEAE cellulose, can pose as a "yardstick" reference chemical to weigh against the efficiency of similar compounds. The applied method is also valuable as a routine tool for the quantitative analysis of very small amounts of chitosan products while depending on the selective ion-exchange capacity of the polymer. The observed linearity and sensitivity of the methods promotes its friendly application in routine analysis of such polymers.

\section{Conclusions}

Simple, quick and inexpensive colorimetric assays serve as convenient means to rapidly and straightforwardly assess product quality in a cost-efficient transaction. The reaction of chitosan with anionic dyes has instantly produced stable and colored adducts. This has triggered developing anionic dyes like bromocresol purple as an analytical reagent for assessing the quality of chitosan. Anionic dyes such as bromocreaol purple and bromocresol green form colored dye-matrix complexes with chitosan to produce a colored product. The dye-binding method produces a colored product which shed and equivalent amount of dye which is linearly proportional to chitosan quantity. Colorimetric tests are rapid and easy to perform. The reagents and equipment for colorimetric tests are inexpensive, environmentally safe, and are ideal for use in non-very well-equipped labs.

\section{References}

[1] M. N. V. R. Kumar, "A Review of Chitin and Chitosan Applications," Reactive and Functional Polymers, Vol. 46, No. 1, 2000, pp. 1-27.
[2] G. A. F. Roberts, "Thirty Years of Progress in Chitin and Chitosan," Progress on Chemistry and Application of Chitin, Vol. 13, 2008.

[3] S.-O. Fernandez-Kim, "Physicochemical and Functional Properties of Crawfish Chitosan as Affected by Different Processing Protocols," M. S. Dissertation, Seoul National University, Seoul, 2004.

[4] N. I. Larionova, D. K. Zubaerova, D. T. Guranda, M. A. Pechyonkin and N.G. Balabushevich, "Colorimetric Assay of Chitosan in Presence of Proteins and Polyelectrolytes by Using O-Phthalaldehyde," Carbohydrate Polymers, Vol. 75, No. 4, 2009, pp. 724-727.

[5] J. J. Park, "Development of Biomems Device and Package for a Spatially Programmable Biomolecule Assembly," Ph.D. Dissertation, Faculty of the Graduate School of the University of Maryland, College Park, 2006.

[6] K. van de Velde and P. Kiekens, "Structure Analysis and Degree of Substitution of Chitin, Chitosan and Dibutyryl Chitin by FT-IR spectroscopy and Solid State ${ }^{13}$ C NMR,' Carbohydrate Polymers, Vol. 58, No. 4, 1998, p. 409.

[7] R. A. A. Muzzarelli, "Colorimetric Determination of Chitosan," Analytical Biochemistry, No. 260, 1998, pp. 255-257.

[8] C. Wischke and H. Borchert, "Increased Sensitivity of Chitosan Determination by a Dye Binding Method," Carbohydrate Research, Vol. 341, No. 18, 2006, pp. 2978-2979.

[9] T. A. Khan, K. K.Peh and H. S. Ch'ng, "Reporting Degree of Deacetylation Values of Chitosan: The Influence of Analytical Methods," Journal of Pharmacy \& Pharmaceutical Sciences, Vol. 5, No. 3, 2002, pp. 205-212.

[10] K. M. Picker-Freyer and D. Brink, "Evaluation of Powder and Tableting Properties of Chitosan," AAPS Pharmaceutical Science and Technology, Vol. 7, No. 3, 2006. 